\title{
Single-cell transcriptomic analysis reveals the critical molecular pattern of UV-induced cutaneous squamous cell carcinoma
}

Guorong Yan $\mathbb{D}^{1,5}$, Liang $\mathrm{Li}^{2,5}$, Sibo Zhu ${ }^{3,5}{ }^{3,5}$, Yuhao Wu${ }^{1}$, Yeqiang Liu ${ }^{4}{ }^{4}$, Lude Zhu ${ }^{1}$, Zijun Zhao ${ }^{1}$, Fei Wu ${ }^{4}$, Ning Jia ${ }^{1}$, Caihe Liao ${ }^{1}$, Long Jiang ${ }^{2}$, Qingyu Zeng ${ }^{1}$, Peiru Wang ${ }^{1}$, Lei Shi ${ }^{1}$, Zhe Zheng ${ }^{1}$, Shan Fang ${ }^{1}$, Guolong Zhang $\mathbb{D}^{1 凶}$, Yichen Tang (iD ${ }^{2 凶}$ and Xiuli Wang (iD ${ }^{\circledR}$

(c) The Author(s) 2021

Cutaneous squamous cell carcinoma (cSCC) is the second most common nonmelanoma skin cancer characterized by high invasiveness, heterogeneity, and mainly occurs in the ultraviolet (UV)-exposed regions of the skin, but its pathogenesis is still unclear. Here, we generated single-cell transcriptome profiles for 350 cells from six primary UV-induced cSCCs, together with matched adjacent skin samples, and three healthy control skin tissues by single-cell RNA-sequencing technology based on Smart-seq2 strategy. A series of bioinformatics analyses and in vitro experiments were used to decipher and validate the critical molecular pattern of cSCC. Results showed that cSCC cells and normal keratinocytes were significantly distinct in gene expression and chromosomal copy number variation. Furthermore, cSCC cells exhibited 18 hallmark pathways of cancer by gene set enrichment analysis. Differential expression analysis demonstrated that many members belonging to S100 gene family, SPRR gene family, and FABP5 were significantly upregulated in CSCC cells. Further experiments confirmed their upregulation and showed that $\$ 100 A 9$ or FABP5 knockdown in CSCC cells inhibited their proliferation and migration through NF-KB pathway. Taken together, our data provide a valuable resource for deciphering the molecular pattern in UV-induced CSCC at a single-cell level and suggest that S100A9 and FABP5 may provide novel targets for therapeutic intervention of CSCC in the future.

Cell Death and Disease (2022)13:23; https://doi.org/10.1038/s41419-021-04477-y

\section{INTRODUCTION}

The impact of cutaneous squamous cell carcinoma (cSCC) in a global context is monstrous and cannot be ignored. According to the lasted global burden of disease study, nonmelanoma skin cancer (NMSC) was the most prevalent cancer in both men and women in 2017, reaching 7.7 million incident cases, of which 5.9 million were caused by basal cell carcinoma and 1.8 million due to CSCC [1]. Furthermore, another recent global cancer statistic research also estimated that there were almost 1.2 million incident cases of CSCC in 2020 [2]. As the second most common NMSC, cSCC develops preferentially in the interfollicular epidermis since the unrestricted proliferation of epidermal keratinocytes (KCs). Cumulative exposure to ultraviolet (UV) radiation and carcinogenic chemicals, the use of immunosuppressive agents, and genetic susceptibility were extensively considered as the major risk factors of CSCC [3]. Although CSCC exhibits a relatively low risk of lymphatic metastasis and good prognosis, there were still $2.1-5 \%$ of CSCCs who progressed into metastases [4, 5], ultimately causing $3900-8800$ deaths in the US [3], accounting for $20 \%$ of cutaneous carcinoma-related deaths [6]. Recently, two approved antiprogrammed death protein-1 (PD-1) antibodies, including
Cemiplimab and Pembrolizumab used for the treatment of locally advanced and metastatic CSCC, are promising. Still, only $34-50 \%$ objective response rate was obtained [7-9], emphasizing the need for the better characterization of CSCC to explore novel therapeutic targets.

Gene expression profiles of tumor tissues have revolutionized our understandings on CSCC [10]. Yet most analyses of gene expression are dominated by bulk RNA-sequencing (RNA-seq) technology, in which the expression profile of each gene is profiled by the average expression of all sequenced cells for each biological sample, masking many critical aspects of intra-tumor heterogeneity (ITH) and hindering our understandings of CSCC biology. What is exciting is that the emergence of single-cell RNA-sequencing (scRNA-seq) technology has revealed major cellular components, heterogeneity of human normal skin, and a wide range of human skin diseases at the single-cell transcriptome level [11], and has been used for the identification of cell type-specific expression quantitative trait loci [12]. Miao et al. found that tumor-initiating stem cells can selectively acquire CD80 by the CSCC mouse model using scRNA-seq, which directly dampen the cytotoxic T cell activity, implicating the vital role of

\footnotetext{
${ }^{1}$ Institute of Photomedicine, Shanghai Skin Disease Hospital, School of Medicine, Tongji University, Shanghai 200092, China. ${ }^{2}$ Department of Dermatologic Surgery, Shanghai Skin Disease Hospital, School of Medicine, Tongji University, Shanghai 200092, China. ${ }^{3}$ State Key Laboratory of Genetic Engineering, School of Life Sciences, Fudan University,

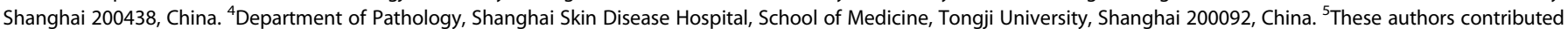
equally: Guorong Yan, Liang Li, Sibo Zhu. ${ }^{凶}$ email: zglamu@163.com; tangyichen@medmail.com.cn; wangxiuli_1400023@tongji.edu.cn Edited by Professor Eleonora Candi
} 
tumor-initiating stem cells in activating the immune checkpoint therapy [13]. Yost et al. performed scRNA-seq in the cSCC patients before and after anti-PD- 1 therapy and observed that $T$ cell response to checkpoint blockade relies on the recruitment of novel T cells [14]. Moreover, a recent study identified a tumorspecific KC population in CSCC using sCRNA-seq, which acted as a hub for intercellular communications [15]. However, most studies employed a 10X Genomics Chromium-based scRNA-seq strategy, which exhibits the advantage in the huge sequenced cell number, but biases the expression quantifications in some key driver genes, when compared it with the Smart-seq2 scRNA-seq strategy. Besides, although the studied tissues were paired for cSCC tissue and adjacent skin from Ji et al., not all CSCC tissues were collected from UV-exposed sites, which may also complicate the interpretation of the result. Furthermore, different ethnic backgrounds tend to possess some specific genetic architectures, and they did not focus on the specific gene expression profile in the cSCC cells. Therefore, it is still vital to decipher the key driver genes of CSCC by a high accuracy scRNAseq technology in different races.

Here, we aimed to adopt the Smart-seq2-based scRNA-seq approach to dissect the CSCC ITH and critical dysregulated genes in promoting CSCC progression. A total of 350 quality-controlled single-cell transcriptomes and 5 cell populations were obtained from six primary human cSCCs and patient-matched skin. A wide range of known and novel dysregulated genes, including the $\mathrm{S} 100$ gene family, SPRR gene family, and FABP5 associated with cSCC progression, were sought out by bioinformatics analyses and validated by a series of in vitro cell experiments. Thus, our findings will update the understanding of the mechanism of UV-induced cSCC progression.

\section{MATERIALS AND METHODS \\ Human patient samples}

Primary CSCC and patient-matched normal adjacent skin samples were obtained from six consecutive CSCC patients ( $86 \pm 6$ years), all of whom were immunotherapy-naive. The detailed clinico-pathological characteristics of these patients are presented in Table 1. All diagnoses of cSCC were verified histologically by a board-certified dermatopathologist (Supplementary Fig. S1), and human papilloma virus-negative. In addition, three more normal young skin tissues were obtained from donors ( $28 \pm 5$ years) who underwent resection surgery, for the investigation of KCs between the young and the old. In specific, all fresh specimens for experiments were collected during surgical resection and immediately stored in RPMI with $10 \%$ fetal bovine serum, $1 \%$ amphotericin $B$, and $1 \%$ penicillin/streptomycin. In addition, paired-samples were also fixed in $10 \%$ formalin solution for downstream immunohistochemistry (IHC) analysis.

\section{Tissue dissociation}

Fresh skin biopsy samples including primary $\mathrm{CSCC}$ and patient-matched normal skin were minced. Each tissue was dissociated in a $2 \mathrm{ml}$ tube (Axygen, China) containing $1 \mathrm{ml}$ pre-warmed M199-media (ThermoFisher Scientific, USA), $2 \mathrm{mg} / \mathrm{ml}$ collagenase P (Roche, USA), and $10 \mathrm{U} / \mu \mathrm{l}$ DNase I (Roche) according to the protocol described by Tirosh et al. [16]. Tissues were digested for $60 \mathrm{~min}$ at $37^{\circ} \mathrm{C}$ and then pipetted up and down every $10 \mathrm{~min}$ for 10 times. After initial isolation, cells were filtered by a $70 \mu \mathrm{m}$ nylon mesh (ThermoFisher Scientific) and were spun at $450 \times \mathrm{g}$ for $5 \mathrm{~min}$ to yield single-cell suspensions. Pellet was resuspended for live cell staining using carboxyfluorescein diacetate succinimidyl ester incubation for $5 \mathrm{~min}$.

\section{Single-cell cDNA library construction and sequencing}

Single cells from each sample were manually and randomly picked by mouth pipette under fluorescent microscopy (X71, Olympus, Japan) and then transferred into $0.2 \mathrm{ml}$ PCR tubes containing $2 \mu \mathrm{l}$ cell lysis buffer. Libraries of isolated single cells were constructed following the Smartseq2 protocol [17] with only modifications on the reverse transcription and amplification cycle.

Oligo-dT primed mRNA reverse transcription was performed with SMARTScribe reverse transcriptase (Takara, Japan) and locked by template-switching oligonucleotides (Exiqon, Denmark). After that, fulllength CDNA amplification was conducted by 22 cycles of PCR amplification with HiFi-HotStart ReadyMix (KAPA Biosystems, USA) and subsequent $0.6 \times$ AMPure beads purification (BD, USA). Barcoded libraries were fragmented and tagmented with Nextera XT Library Prep kit (Illumina). Pooled libraries with unique N5-N7 barcodes were sequenced using a Hiseq 2500 platform (Illumina) with a single-end 50 base-pair length.

\section{scRNA-seq data processing}

Raw fastq data of scRNA-seq were first assessed by FastQC (v0.11.9) [18]. Then, the reads with the adaptor or poly-A sequences were removed from the raw fastq data before alignment using Trimmomatic (v0.36) [19]. The low-quality reads with " $\mathrm{N}$ " bases rate $>0.05$, average quality $<20$, and the read length $<30 \mathrm{bp}$ were also removed. Second, clean reads were aligned to the UCSC human reference genome hg19 using HiSat2 (v2.0.5) [20]. We used FeatureCounts [21] to count the number of uniquely mapped read pairs for each gene. We defined gene with count $>1$ as the detected gene. Low-quality cells $(<1000$ genes/cell, $<10,000$ library size/cell, and $>10 \%$ mitochondrial genes) were excluded. Then, we normalized the gene expression by computerSumFactors and logNormCounts functions using scran R-package [22].

\section{Cell type identification}

The Seurat R-package was employed to identify the major cell types [23]. A total of 2000 highly variable genes were sought out using the FindVariableGenes function and then used to perform principal component analysis (PCA) for the dimensionality reduction. Significant principal components (PCs) were identified using the JackStraw function. PCs 1-14 were used for graph-based clustering (resolution $=1$ ) to identify distinct clusters. These clusters were visualized by t-distributed stochastic neighbor embedding (t-SNE) analysis using previously computed PCs $1-14$. The cell markers were determined by the FindAllMarkers function with 0.1 and 0.25 for parameters of min.pct and logfc.threshold, respectively. Then, we annotated the identity of each cell cluster based on the expression of known markers: KRT15, KRT7 (normal KCs), CFD, APOD (fibroblasts (FBs)), CDKN2A, KRT6A (cSCCs), CD79A, IGLL1, JCHAIN, MZB1 (B cells), and RGS1, TYROBP (dendritic cells (DCs)).

\section{CNV inference}

To investigate the copy number variation (CNV) level in normal KCs and CSCC cells, we calculated CNVs in cluster 0 and cluster 2 based on the original gene count data of scRNA-seq data using inferCNV R-package (v.1.2.1). The normal KCs from cluster 0 were calculated as a CNV control to eliminate the individual somatic CNV. The CNV score was computed on a

Table 1. Clinico-pathological characteristics of the patient cohort.

\begin{tabular}{|c|c|c|c|c|c|c|}
\hline Patient & Gender & Age (years) & Location & Differentiation & Immune-infiltration & TNM stage \\
\hline 1 & $\mathrm{~F}$ & 85 & Right cheek & Well differentiated & High & T1cN0M0 \\
\hline 2 & $\mathrm{~F}$ & 76 & Forehead & Well differentiated & Moderate & T1CNOMO \\
\hline 3 & M & 89 & Crown of scalp & Moderately differentiated & High & T3NOMO \\
\hline 4 & $\mathrm{~F}$ & 88 & Left cheek & Moderately differentiated & High & T1bNOMO \\
\hline 5 & $\mathrm{~F}$ & 91 & Right cheek & Well differentiated & High & T3N1M0 \\
\hline 6 & M & 91 & Right temple & Well differentiated & High & TisNOMO \\
\hline
\end{tabular}


moving average window equal to 101 , and the cutoff parameter was set to 1. The scores were restricted to the range of -1 to 1 by replacing all values $>1$ with 1 and all values $<-1$ with -1 .

DEGs identification and function annotation

To detect the differentially expressed genes (DEGs) between KCs and cSCC cells, we used the FindMarkers function implemented in Seurat R-package. The threshold for DEGs detection was as follows: the absolute value of average $\log F C$ was set to 1 and the adjusted $P$ value was set to 0.05 . Then, Gene Ontology (GO) analysis at the biological process level, Kyoto Encyclopedia of Genes and Genomes (KEGG) analysis, and protein-protein interaction (PPI) network analysis were performed in the STRING website (https://www.string-db.org). Gene set enrichment analysis (GSEA) was conducted using clusterProfiler R-package.

\section{TCGA database analysis}

In order to further investigate the expressions of S100A9, SPRR2A, and FABP5 among different SCCs, we used the RNA-seq data including cervical squamous cell carcinoma and endocervical adenocarcinoma (CESC), esophageal carcinoma (ESCA), head and neck squamous cell carcinoma (HNSC), and lung squamous cell carcinoma (LUSC) from The Cancer Genome Atlas (TCGA) database. Correlations among S100A9, $S P R R 2 A$, and FABP5 were also investigated. Finally, relationships between survival times and these genes were analyzed by GEPIA tools (http:// gepia2.cancer-pku.cn/\#index).

\section{qRT-PCR analysis}

Primary CSCCs and patient-matched normal adjacent skin tissues for quantitative real-time PCR (qRT-PCR) were obtained from consecutive CSCC patients. Total RNA was extracted from CSCC tissues and paired adjacent normal skin tissue using Trizol reagent (Invitrogen, USA). Then, extracted RNAs were reverse-transcribed into CDNA and were then subjected to Taqman qPCR analysis on a 7900HT Fast RT-PCR System (Life Technologies, ThermoFisher, Loughborough, UK). The mRNA levels of S100A9, SPRR2A, and FABP5 were detected by the SYBR Green qPCR system (Life Technologies) using the following primers (Supplementary Table S1). GAPDH mRNA level was used as a control. The relative expressions were calculated with $2^{-\triangle \Delta C T}$ method.

\section{Immunohistochemistry staining}

The detailed procedure for IHC staining was presented in our previous published study [24]. Briefly, samples were fixed in $10 \%$ neutral buffered formalin, dehydrated with gradient ethanol, hyalinized in xylene, embedded in paraffin, and cut into sections with a thickness of $5 \mu \mathrm{m}$. Sections were incubated with primary antibodies: anti-S100A9 (Abcam, \#ab92507, 1:250) and anti-FABP5 (CST, \#39926, 1:1200). The temperature was maintained at $4{ }^{\circ} \mathrm{C}$ overnight, followed by the co-incubation of secondary antibody for $1 \mathrm{~h}$ after washing with phosphate-buffered saline. Finally, sections were immunostained using DAB plus kit.

\section{Cell culture and transfection}

The human epidermoid carcinoma A431 cell line from the National Infrastructure of Cell Line Resource and SCL-1 cell line (Daixuan Biotech, Shanghai, China) were confirmed by the GENEWIZ commercial company (Suzhou, China). Then cells were seeded in cell culture dishes and cultured for 3 days till confluency at 90\%. Small interfering RNA (siRNA) were provided by RIBOBIO (Guangdong, China). The small interfering negative control RNA (siNC) was nonhomologous to any human genome sequences. The siRNA-targeted sequences are present in Supplementary Table S2. All transfections were performed with Lipofectamine 3000 Transfection Reagent (Invitrogen) according to the manufacturer's direction.

\section{CCK-8 assay}

According to the standard manufacturer's protocol, cell viability in different groups was tested by CCK- 8 assay (Dojindo, Japan). A density of $5 \times 10^{3}$ cells/well was seeded in 96 -well plates and incubated at $37^{\circ} \mathrm{C}$ for different times $(24,48$, and $72 \mathrm{~h})$. Before testing, $10 \mu \mathrm{l}$ of CCK- 8 reagent was added to each well and for a $4 \mathrm{~h}$ incubation. After that, the absorbance (optical density) of cells was captured and recorded at $450 \mathrm{~nm}$ using a Varioskan Flash system (Thermo, USA)

\section{Cell apoptosis assay}

Flow cytometer assay was used for detecting cell apoptosis. Briefly, cells were resuspended in fixation buffer and stained with $5 \mu \mathrm{l}$ Annexin V/FITC and $5 \mu \mathrm{l}$ propidium iodide (PI) solutions for $30 \mathrm{~min}$, respectively. Then cells were analyzed by flow cytometry (BD Biosciences, USA) with FITC and PI channel.

\section{Wound-healing assay}

Different groups of transfected cells were seeded in a 24-well plate and grew until almost $90 \%$ confluence. A $200 \mu$ l pipette tip was used to scratch a line on the cell layer. The movement of cells was captured by an Olympus microscope at 0 and $24 \mathrm{~h}$, respectively. The mean distance of each wound was calculated by the Image J software.

\section{Western blot analysis}

To investigate whether NF-KB pathway involving the CSCC progression, western blot (WB) analysis was performed to observe its change after S100A9 or FABP5 knockdown. The total protein was extracted from A431 and SCL-1 cell lines with RIPA buffer, and the concentrations were determined by a BCA protein assay kit (EpiZyme Biotechnology, Shanghai, China). Briefly, the equal amount of protein for each sample was separated by $12 \%$ SDS-polyacrylamide gels and transferred to the polyvinylidene difluoride membranes, which was then blocked in $5 \%$ skim milk for $1 \mathrm{~h}$ at room temperature, followed by incubating with the primary antibodies at $4{ }^{\circ} \mathrm{C}$ for one night. Antibodies against p65 (CST, \#8242), p-p65 (CST, \#3033), and GAPDH (CST, \#5174) were diluted by 1:1000. After three times washing with tris-buffered saline tween buffer, the membranes were incubated with the secondary antibodies at room temperature for $1 \mathrm{~h}$. Finally, the protein bands were visualized by the chemiluminescence method.

\section{Statistical analysis}

Data were presented as the mean \pm standard deviation (SD). Data were statistically analyzed using Prim 9. Statistical significance was determined by paired or non-paired Student's $t$-test. $P<0.05$ was considered statistically significant.

\section{RESULTS \\ Single-cell transcriptomes of cSCC}

Six CSCC tissues, paired adjacent skin, and three healthy control skin tissues were collected for scRNA-seq based on the Smart-seq2 strategy (Fig. 1A). After quality control, a total of 350 cells with an average of 6319 genes/cells were obtained for the following analyses. Then, five cell clusters consisting of normal KCs (112 cells), FBs (99 cells), cSCCs (75 cells), B cells (38 cells), and DCs (26 cells) after variable features selection, PCA and PCs selection (Fig. 1B-D). In general, most cells of the adjacent skin samples and healthy control skin samples were clustered into $\mathrm{KC}$ cluster and most cells of the CSCC tissues were clustered into CSCC cells and immune cells (Fig. 1D). Notably, three KCs from the cSCC adjacent tissues were clustered into the CSCCs cluster. Furthermore, no significant difference of KCs between the young and the old was found as they clustered into one cluster. Cluster-specific markers were identified to annotate cell populations. In most cases, widely used cell type markers were used for cell type definition. The cell markers are as follows: KCs: KRT15, KRT7; FBs: CFD, APOD; cSCC cells: CDKN2A, KRT6A; B cells: CD79A, IGLL1, JCHAIN, MZB1; DCs: RGS1, TYROBP (Fig. 1C).

\section{CNV landscape of cSCC cells}

CNV analysis was often widely used to distinguish malignant from non-malignant cells. To investigate the malignancy of CSCC cells, we calculated large-scale chromosomal CNV in normal KC and CSCC cell clusters based on averaged expression patterns across intervals of the genome. We found that CSCC cells exhibited remarkably higher CNV levels than Normal KC (Fig. 1E). Specifically, most CSCC cells showed higher CNV levels on chromosomes 1, 3, 4, 6,9 , and 17. However, there were also some CSCC cells that showed low CNV scores. 
A
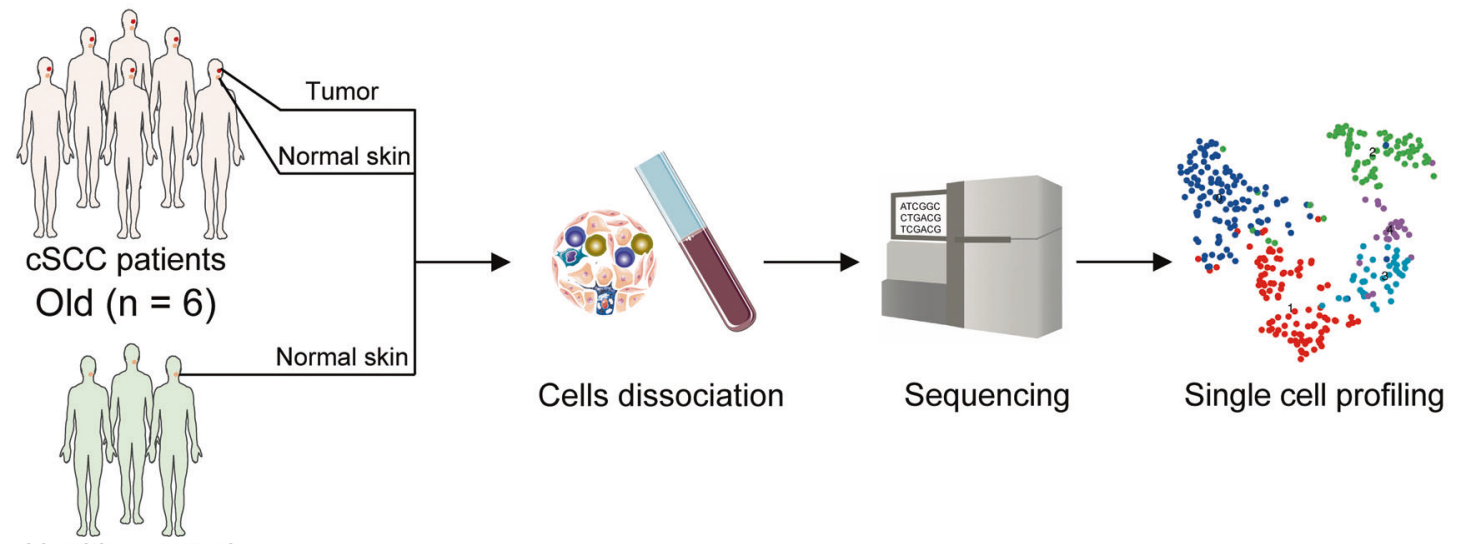

Cells dissociation

Sequencing

Single cell profiling

Healthy control

Young $(n=3)$

B

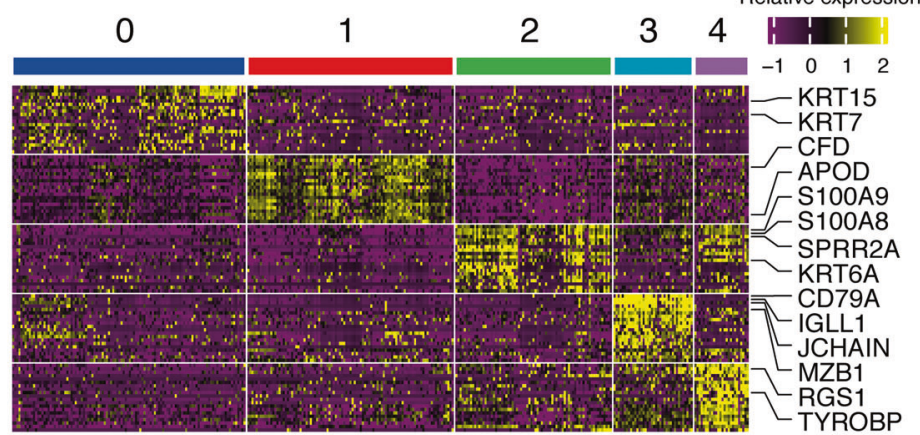

C

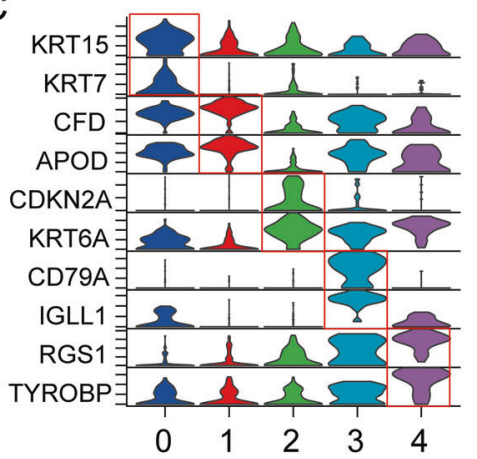

D

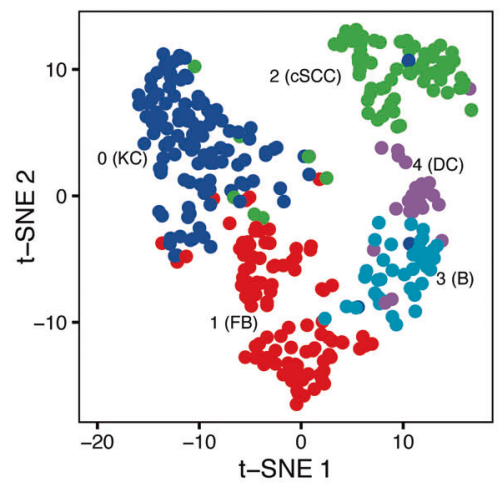

E

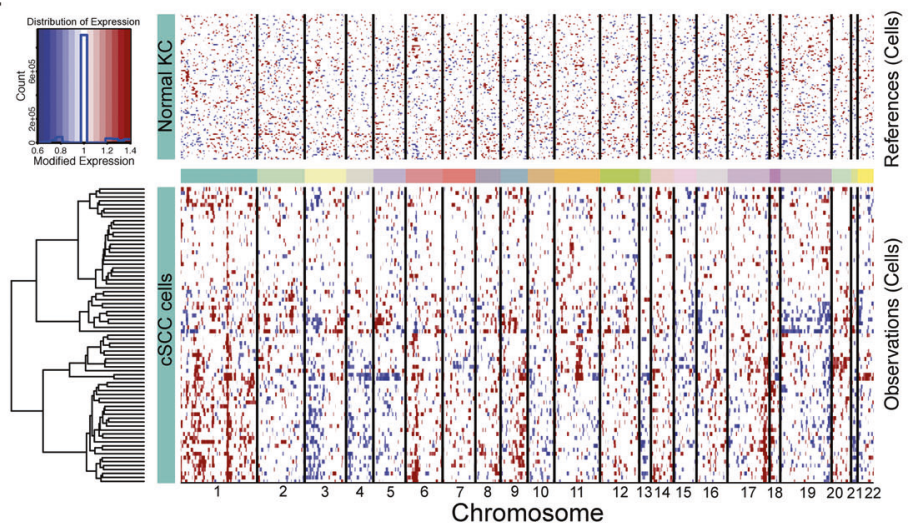

Fig. 1 ScRNA-seq profiling of the cSCC tumor environments. A Workflow of cSCC patients and healthy control sample processing for scRNAseq. B Heatmap showing the expression of representative genes in each cluster. The top bars label the clusters corresponding to specific cell clusters. C Violin plots displaying the expression of well-known representative markers across the cell types identified in cSCC. D T-distributed stochastic neighbor embedding (t-SNE) plot shows the annotation for cell types. E Heatmap showing large-scale CNVs of normal KC and cSCC cells. The normalized CNV levels were shown, the red color indicates high CNV level and blue indicates low CNV level.

\section{Dysregulated genes and pathways in CSCC}

In order to identify the DEGs between CSCC and normal KCs at a single-cell level, we performed differential expression analysis between KCs (Cluster 0 ) and CSCCs (Cluster 2) cells. A total of 161 DEGs were identified including 119 upregulated and 42 downregulated genes in CSCC (adjusted $P$ value $<0.05$ and $|\log F C|>1$ ), which indicates that most of DEGs were upregulated in CSCC (Fig. 2A). The top 10 significantly upregulated genes were highlighted, including S100A9, S100A8, SFN, S100A7, S100A2, SPRR2A, FABP5, ISG15, KRT6B, and KRT16. In specific, seven keratin encoded genes (KRT5, KRT6A, KRT6B, KRT6C, KRT14, $K R T 16$, and KRT17), six genes (S100A2, S100A7, S100A7A, S100A8, and S100A9) from the $S 100$ family, and five genes (SPRR2A, $S P R R 2 B, S P R R 2 D, S P R R 2 F$, and SPRR1B) from SPRR family were significantly upregulated in CSCC cells, indicating the pivotal function of these gene families in CSCC progression. We also validated three upregulated genes including $S 100 A 9, F A B P 5$, and $S P R R 2 A$, and three downregulated genes including CFD, APOD, and VIM by two external independent studies including one scRNA-seq study by $10 X$ Genomics platform (GSE144240) and one bulk RNA-seq study (GSE108010). In general, the upregulation of S100A9 and FABP5, and the downregulation of CFD, $A P O D$, and VIM were validated in both public sCRNA-seq study and bulk RNA-seq study, although the significant difference was 

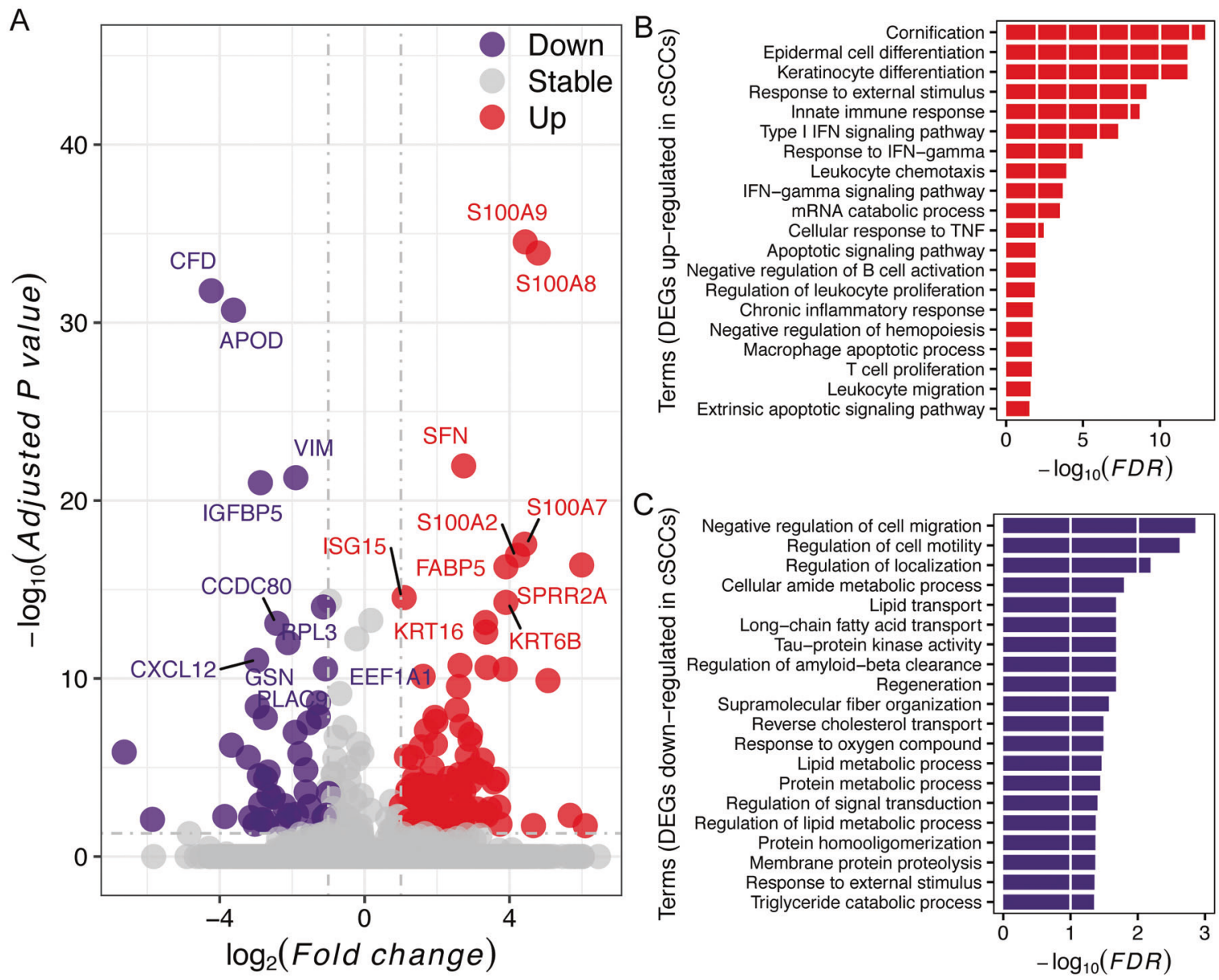

Fig. 2 Dysregulated genes and biological processes in cSCC. A Volcano plot for DEGs identification between KCs and cSCCs. B Biological processes annotated by upregulated genes in CSCCs. C Biological processes annotated by downregulated genes in cSCCs.

not detected in all patients from the public scRNA-seq study (Supplementary Fig. S2). However, the upregulation of SPRR2A was only identified in one donor (patient 4) from the public scRNA-seq study (Supplementary Fig. S2A), and no difference was found in the bulk RNA-seq dataset (Supplementary Fig. S2B). The further qRT-PCR analysis also confirmed their expression (Supplementary Fig. S3).

GO annotation at the biological process level showed that most upregulated DEGs were annotated to immune responses such as, leukocyte chemotaxis, proliferation, and migration, together with the response to type I IFN, IFN- $\gamma$ signaling pathway, and TNF signaling pathway (Fig. 2B). However, most downregulated DEGs were annotated to lipids metabolism (Fig. 2C). KEGG functional annotation showed that 12 significantly enriched pathways were identified (Supplementary Table S3), most of which were inflammation-related, i.e., IL-17 signaling pathway, and its downstream chemokine signaling and TNF signaling pathway.

\section{PPI network and GSEA analysis}

Pathway annotation can reveal the related pathways in which the DEGs are involved, and PPI network analysis can demonstrate the potential interaction relationship. We found that most of the DEGs interact with many genes (Fig. $3 \mathrm{~A}$ ). Highly mutated oncogene CDKN2A interacted with HIF1A, which interacts with CXCR4. Meanwhile, FABP5 connects with FABP4, which also interacted with CDKN2A by CEBPB. Another network is constructed by SPRR and KRT gene families. GSEA showed that 18 hallmarks of cancer were significantly enriched in CSCCs (Fig. 3B). Taken together, the
S100 gene family, SPRR gene family, and FABP5 may involve in CSCC progression.

Positive correlation among S100A9, SPRR2A, and FABP5

As there is no CSCC-related RNA-seq data in the TCGA database, we investigated the expressions of S100A9, SPRR2A, and FABP5 in four different kinds of SCCs including CESC, ESCA, HNSC, and LUSC. In general, these three genes were upregulated in CESC, ESCA, and LUSC (Fig. 4A). However, S100A9 and SPRR2A were significantly downregulated in HNSC, and the expressions of S100A9 and SPRR2A are very high in both HNSC tissues and normal tissue (Fig. 4A). Although the upregulation and downregulation of these genes in different SCCs should be further investigated, the positive correlations between each other are very high (Fig. 4B and Supplementary Fig. S4), indicating a tight relationship among these three genes. However, no significant effect on survival time was observed.

\section{S100A9, SPRR2A, and FABP5 were overexpressed in CSCC} tissues

For the potential importance of S100, SPRR, and FABP5 in CSCC progression, we further validated the expression of $S 100 A 9$, SPRR2A, and FABP5 by qRT-PCR and IHC in CSCC tissues, matched adjacent skin tissues, and normal skin tissues from healthy donors. qRT-PCR results showed that both of these three genes were significantly overexpressed in CSCC tissues at the mRNA level ( $P<0.001$, Fig. 5 and Supplementary Fig. S5), and there was no significant difference between healthy donors and adjacent skin tissues from CSCC (Fig. 5A, C). Since no 

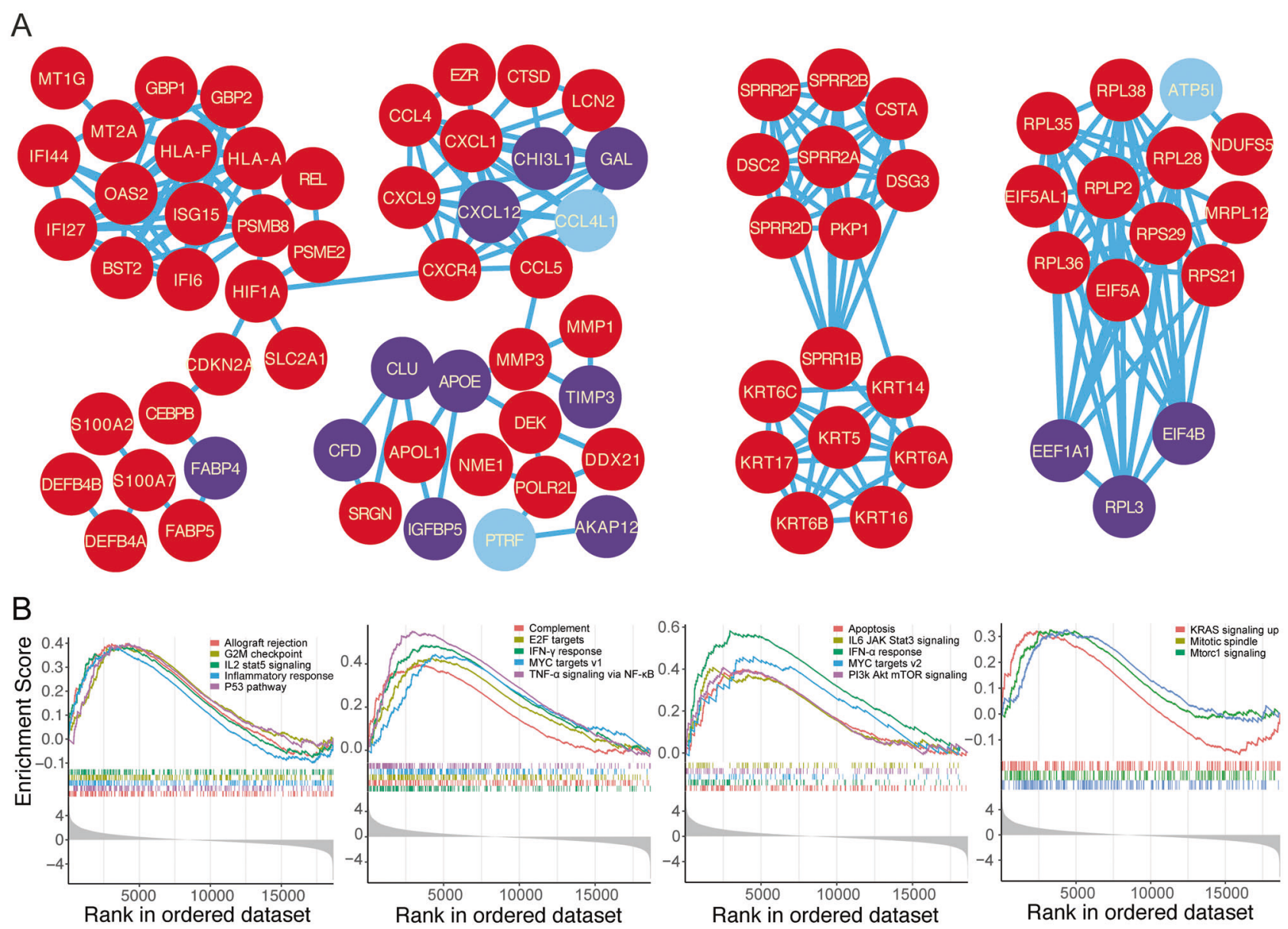

Fig. 3 PPI network and GSEA analysis. A PPI network analysis shows the interactions among the DEGs. B Many cancer-related pathways were upregulated in CSCCs by GSEA.

significant effect on cell migration of SPRR2A in both A431 and SCL-1 cell lines, we did not validate its expression by IHC. Thus, the high expressions of $5100 A 9$ and FABP5 in CSCC tissue were confirmed by IHC (Fig. 5B, D). Collectively, these data further validate the expression of S100A9 and FABP5 in the mRNA and protein levels.

S100A9 and FABP5 promoted cell proliferation and migration To test whether S100A9, SPRR2A, and FABP5 could contribute to the human CSCC cells progression, we investigated these genes on the migration and invasion effect of A431 and SCL-1 cell lines by knocking down their expressions using siRNA. These three genes were knocked down successfully confirmed by qRT-PCR $(P<0.05)$. CCK-8 assay showed that the ability of cell proliferation was significantly inhibited after $24 \mathrm{~h}$ when S100A9 was interfered with siRNA ( $P<0.05$, Fig. 6). However, no significant apoptosis change was observed by the FACS instrument (Fig. 6). Regarding FABP5, we observed a significant decrease in cell proliferation (Fig. 6) and a significant increase of cell apoptosis (Fig. 6). No significant effect of SPRR2A on cell proliferation was observed (Supplementary Fig. S6). Furthermore, both S100A9 and FABP5 siRNAs groups exhibited a significant decrease in migration (Fig. 7A, B), while no significant decrease was found in the SPRR2A group (Supplementary Fig. S7). WB analysis showed that p-p65 expression was significantly decreased after $\$ 100 A 9$ or FABP5 knockdown $(P<0.01$, Fig. $7 C)$, indicating that NF-KB pathway may involve the $C S C C$ progression supervised by S100A9 and FABP5.

\section{DISCUSSION}

cSCC serves as the second most common NMSC, caused by genetic mutations that result from endogenous and environmental factors, such as cumulative UV radiation. Numerous biological or genetic differences of tumor cells result in the cancer ITH [25], which plays a vital role in tumor progression and drug resistance [26]. Thus, it is important to thoroughly demonstrate the gene expression pattern of individual cells [27]. Single-cell transcriptome technology overcomes the disadvantages of traditional bulk RNA-seq methods, by profiling the whole-transcriptome profiles at a single-cell resolution and annotating different cell types from the tumor tissue [28]. Moreover, scRNA-seq will facilitate a clearer understanding of the molecular mechanisms in promoting tumor occurrence, and reveal the somatic mutations during tumor evolution [29]. In the present study, six primary CSCCs, patient-matched adjacent skin samples, and three healthy control skin tissues were used for scRNA-seq to decipherer the ITH of CSCC and driver genes in CSCC progression. A total of 350 high-quality cells were obtained, and five cell clusters were annotated after quality control. Regarding the normal $\mathrm{KCs}$, there was no significant difference between the young and the old. However, three KCs from the cSCC adjacent tissues were also clustered into the cSCC cluster. In the present study, the cSCC patients were old, who undergo long-term UV radiation and whose adjacent skin was also aging. Thus, some KCs from the aged and UV-radiated adjacent or nonadjacent skin tends to harbor genetic mutation or epigenetic modifications, such as TP53, although the skin seems to be 

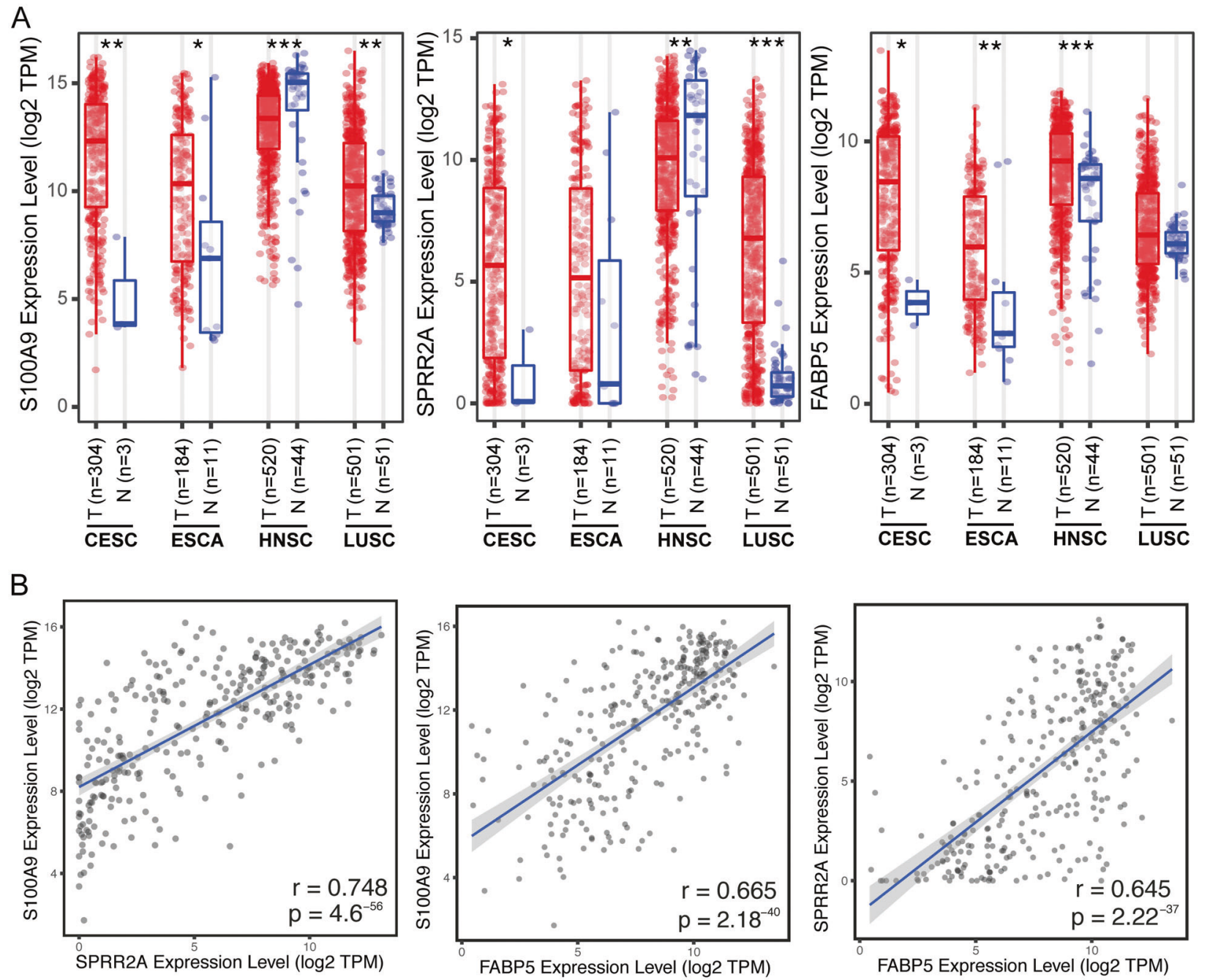

Fig. 4 Expressions of S100A9, SPRR2A, and FABP5 among different SCCs. A S100A9, SPRR2A, and FABP5 expressions in CESC, ESCA, HNSC, and LUSC. B Spearman's rank correlation between each other in CESC. cervical squamous cell carcinoma and endocervical adenocarcinoma for CESC, esophageal carcinoma for ESCA, head and neck squamous cell carcinoma for HNSC, and lung squamous cell carcinoma for LSCC. ${ }^{*} P<0.05,{ }^{*} P<0.01$, and ${ }^{* *} P<0.001$.

apparently normal [30]. Further CNV-based analysis confirmed the malignant of CSCC cells, as higher copy numbers were observed in CSCC cells.

Compared with the normal KCs, 119 upregulated and 42 downregulated genes were sought out in CSCC cells. Notably, many DEGs consisted of S100, SPRR gene family, including S100A2, S100A7, S100A7A, S100A8, S100A9, SPRR2A, SPRR2B, $S P R R 2 D, S P R R 2 F$, and SPRR1B were identified. In humans, the S100 protein family is composed of 21 members that exhibit a high degree of structural similarity but are not functionally interchangeable [31]. S100A8 and S100A9 are thought to be characteristic of several acute and chronic inflammatory disease, including psoriasis, inflammatory bowel disease, and rheumatoid arthritis [32]. Furthermore, S100A8 and S100A9 are upregulated in a variety of cancers such as lung cancer, prostate cancer, colon cancer, stomach cancer, and breast cancer [33]. However, decreased expression of S100A8 and S100A9 in Chinese esophageal squamous cell carcinoma were also reported [34]. Recent studies have shown that S100A8/A9 was highly expressed in CSCC than that in normal and actinic keratosis tissues, and in vitro experiments confirmed that the overexpression of S100A8/A9 could enhance the proliferation and invasiveness of $\operatorname{cSCC}[35,36]$. In the present study, we also detected overexpression of S100A9 in CSCC tissues and validated it by two public datasets, qRT-PCR and IHC. S100A9 knockdown inhibited the proliferation and migration of CSCC cell lines. However, there was no significant effect on cell apoptosis when downregulated S100A9. Regarding the involved pathways, studies have shown that S100A8/A9 induces the activation of the NF-KB pathway, which increases the expression of $C X C L 1$, CCL5, CCL7, and so on, whose products are known in angiogenesis, tumor migration, wound healing, and the formation of pre-metastatic niches into the surrounding tissue and organs [37]. We also observed the decrease of p-p65 after S100A9 knockdown, which confirmed the NF-kB pathway in cSCC progression. Taken together, the $\mathrm{S} 100$ gene family, at least S100A9, played an important role in promoting CSCC progression, including proliferation and migration.

SPRR2A is one of SPRR gene that encodes for a skin cross-linking protein, which plays an important role in maintaining the structural integrity of the epidermal cornified cell envelope [38]. In the normal status, SPRR2A shows the highest expression in the cervix and esophagus, but with low or absent expression in mesenchymal tissues such as brain, heart, muscle, and adipose [39]. Besides, SPRR2A is dominantly overexpressed under inflammation, stress, and infection statuses to protect barrier epithelial 
A

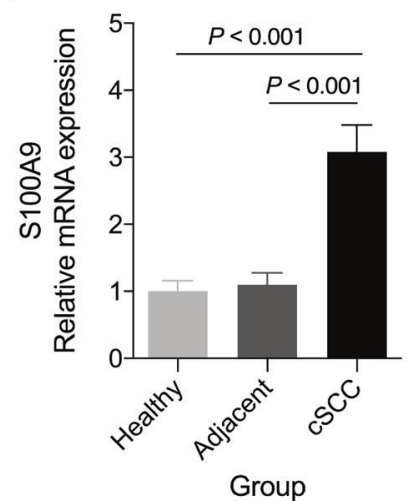

C

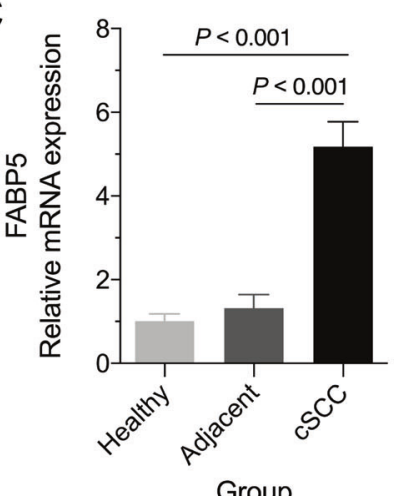

B
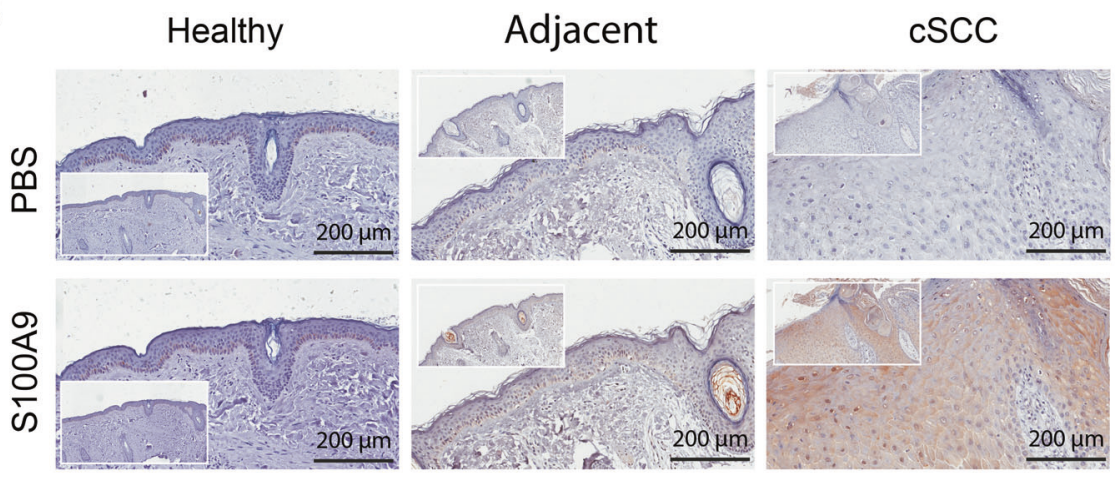

$200 \mu \mathrm{m}$

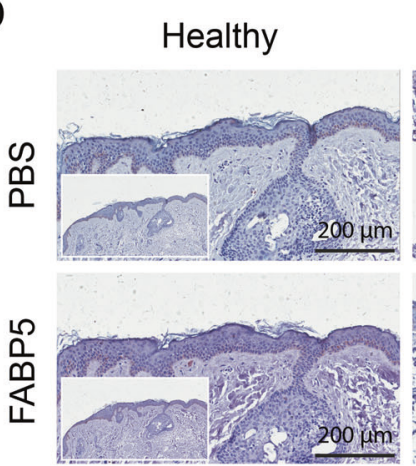

Adjacent

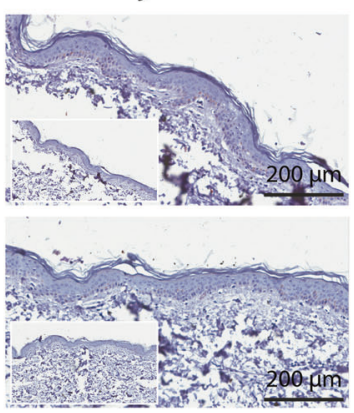

cSCC

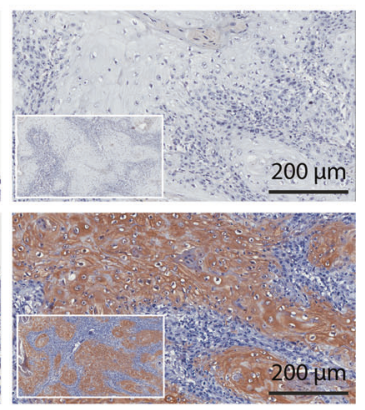

Fig. 5 S100A9 and FABP5 were highly expressed in cSCC tissue. A, B S100A9 was upregulated in cSCC tumor tissue at both mRNA level and protein level by qRT-PCR and IHC. C, D FABP5 was upregulated in CSCC tumor tissue at both mRNA level and protein level by qRT-PCR and IHC. The statistical method is the paired Student's $t$-test for the qRT-PCR results.

cells of the skin, lung, and intestine $[40,41]$. Until now, almost all studies focused on the cell process of SPRR2A-induced epithelialmesenchymal transition (EMT), including embryogenesis, wound healing, and carcinogenesis [42, 43]. Although SPRR2A is commonly expressed in organs containing squamous epithelia, its expression mode is distinct among different SCCs. Specifically, SPRR2A is upregulated in LUSC [44], and HNSC [45], but downregulated in oral SCC [46], and ESCA [47]. Furthermore, the expression of SPRR2A is upregulated first, and then decreases with carcinoma progression (higher stage and lymphatic metastasis) in LUSC and HNSC $[44,45]$. In the present study, we observed the overexpression of SPRR2A in primary CSCC cells and almost absent expression in normal $\mathrm{KC}$, which is consistent with the results in LUSC and HNSCC. With regard to other cancers, SPRR2A is upregulated in gastric cancer [48], but downregulated in cholangiocarcinoma [49]. In the cholangiocarcinoma and liver cancer model, SPRR2A is activated by STAT-3, promoting EMT through the interaction with ZEB1 $[39,49]$. In addition, SPRR2A can increase P53 deacetylation by impairing P300-P53 interactions to inhibit P53 transcriptional targets, accelerating EMT and wound healing [50]. Although down- and upregulation of SPRR2A were observed in different cancers, higher expression of SPRR2A will increase the local aggressiveness of cancers. In contrast, when cancers progress into poor differentiation or lymphatic metastasis, the expression of SPRR2A will decrease to give rise to metastases $[44,45,49]$.

Dysregulated lipid metabolism is the most prominent metabolic alteration in various cancers, playing a vital role in their proliferation, invasion, metastasis, and response to different therapies [51]. FABP5, also known as epidermal FABP (E-FABP), is essential for KC homeostasis and differentiation, and maintenance of the skin-water permeability barrier by participating in fatty acid metabolism $[52,53]$. In the current study, we identified FABP5 was significantly upregulated in CSCC cells compared with the normal $\mathrm{KCs}$, which is consistent with two studies that also confirmed the overexpression of FABP5 by IHC in CSCC $[54,55]$. After then, the same result was observed in actinic keratosis and Bowen's disease [56], which are considered as two premalignant lesions of cSCC. Although many studies have reported the dysregulation of FABP5 in CSCC or its premalignant lesions, no further experiment was conducted to elucidate its mechanism. Here, we found FABP5 can promote cell proliferation, migration, and inhibit apoptosis by in vitro experiment, which was also reported in oral SCC [57]. Besides, FABP5 promotes lymphatic metastasis in cervical cancer, prostate cancer progression, breast cancer progression, and KC differentiation by reprogramming fatty acid metabolism through the NF-KB signaling pathway $[52,58,59]$. Here, we also confirmed the suppression of NF-KB pathway after FABP5 knockdown, underlining the importance of NF-KB pathway in CSCC progression again. In hepatocellular carcinoma, the upregulation of FABP5 enhances hypoxia-inducible factor-1 alpha (HIF-1a) activity, causing lipid metabolism reprogramming, and carcinoma progression [60]. In addition, FABP5 can capture S100A7 from cytosolic psoriatic protein extracts to form the FABP5-S100A7 complex, and vice versa by overlay assays [61]. Collectively, FABP5 is upregulated in various cancers, which promotes cancers progression or metastasis by reprogramming fatty acid metabolism through the NF-KB signaling pathway or enhancing HIF-1a activity. The specific mechanism of FABP5 and NF-KB pathway in CSCC should be further investigated. 
A

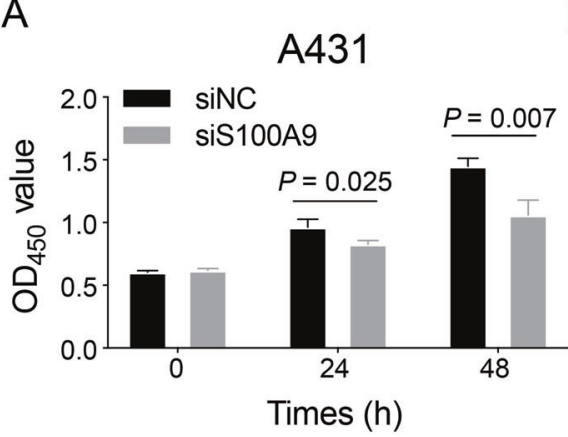

C

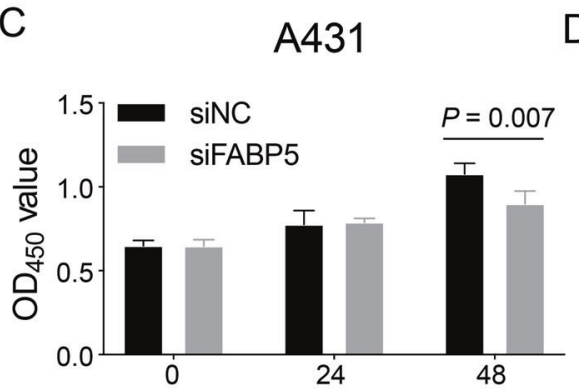

Times (h)
B

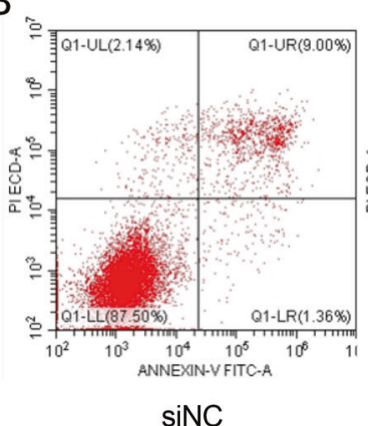

siNC

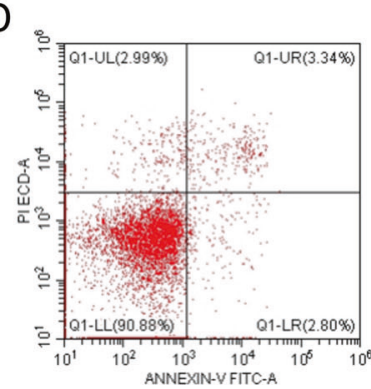

siNC

\section{F}

E

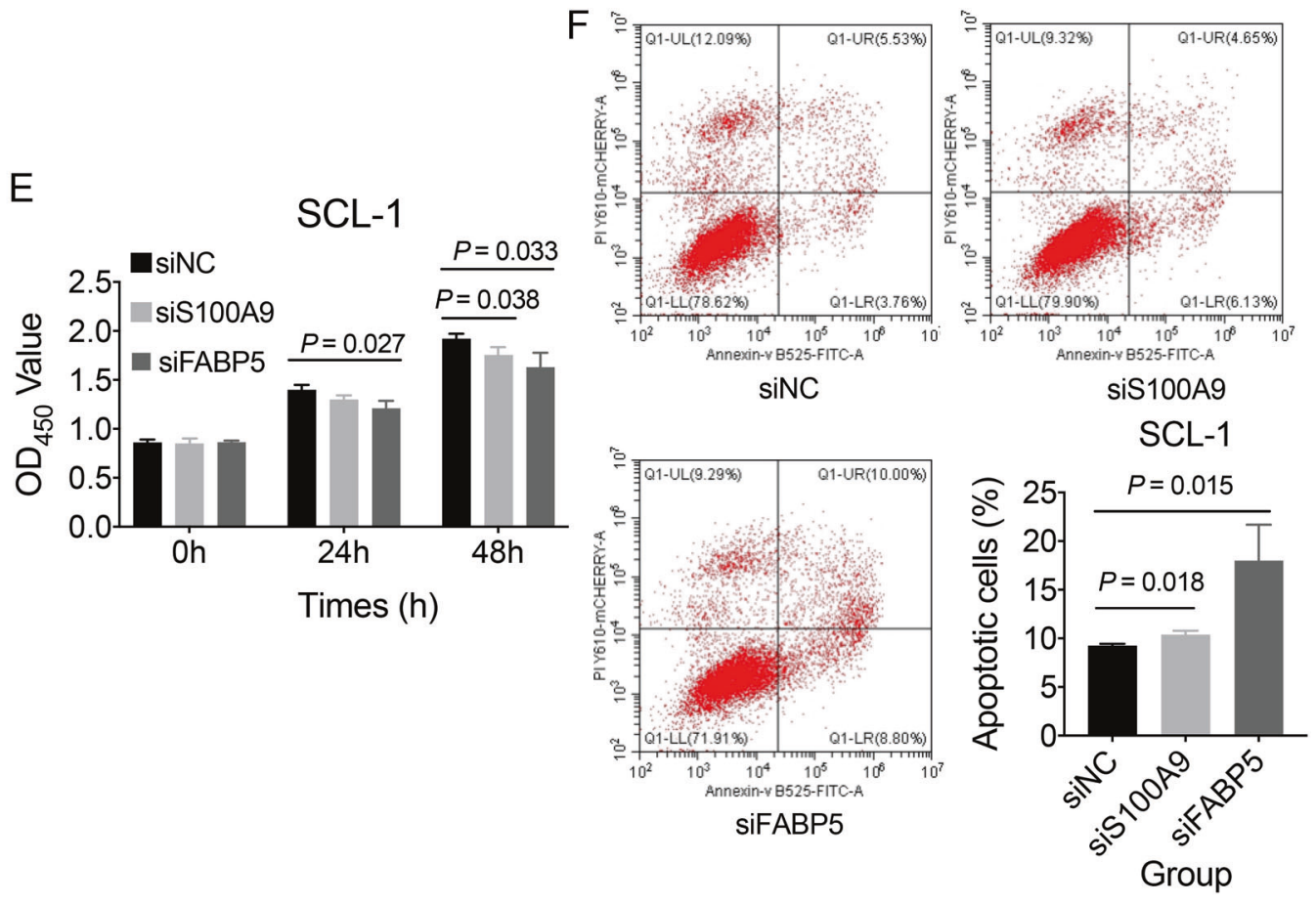

SiNC
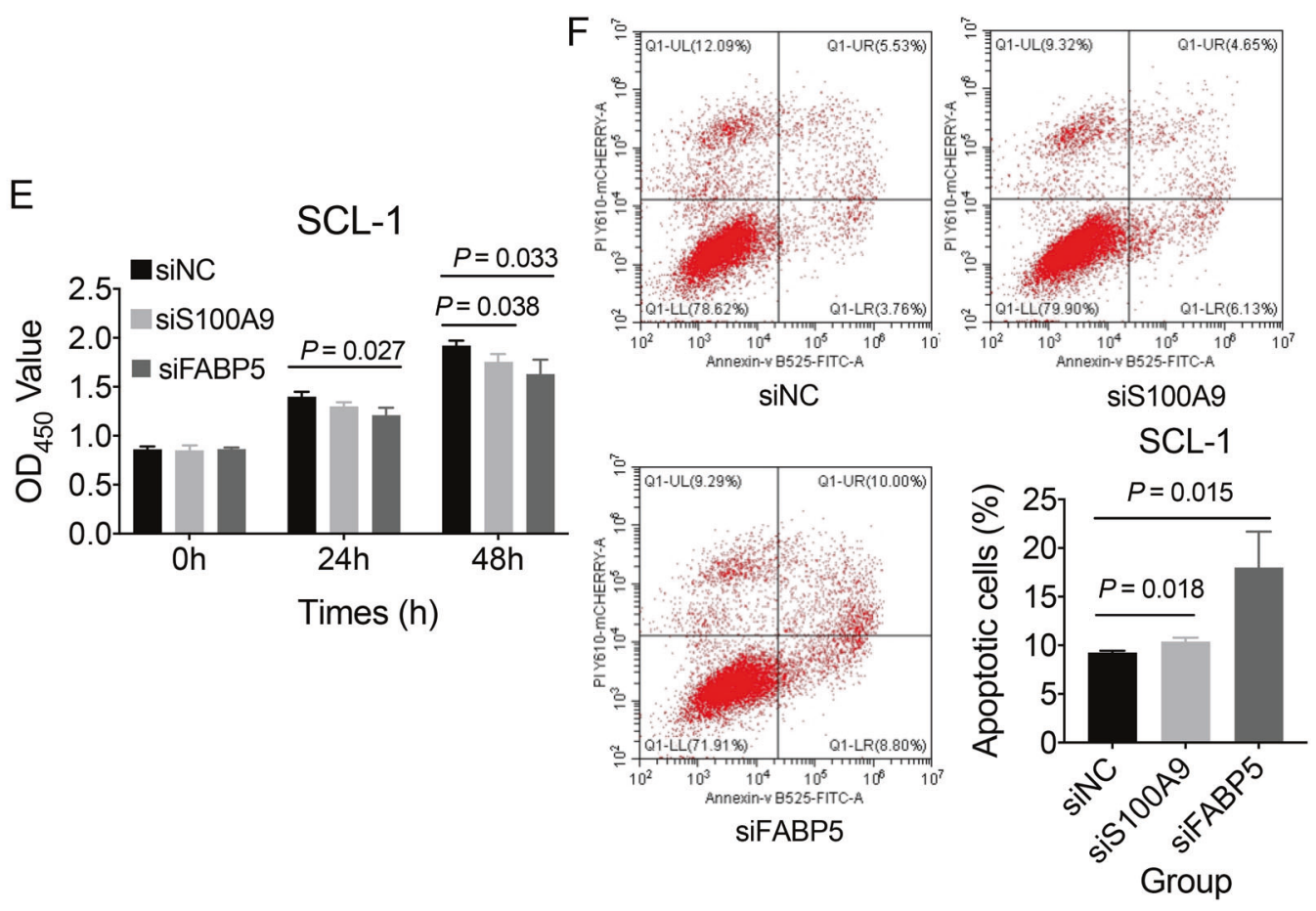
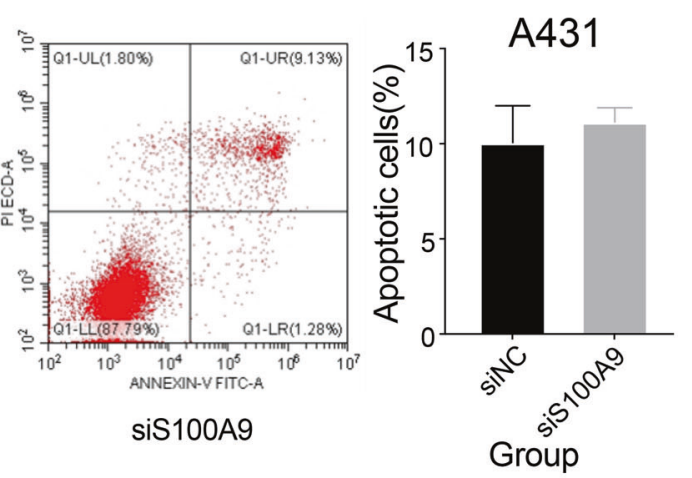

A431

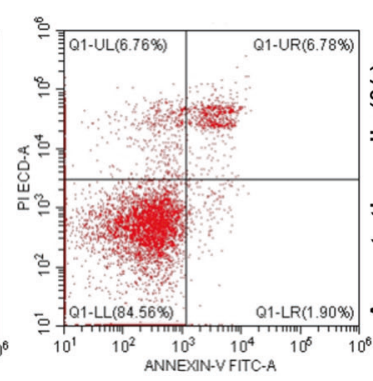

SiFABP5

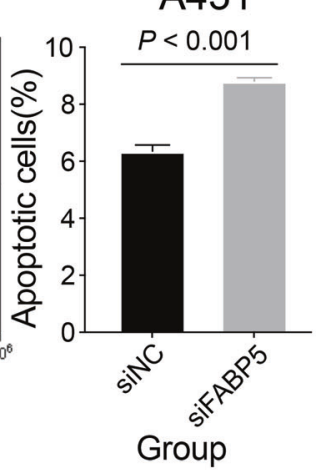

Fig. 6 Effect of S100A9 and FABP5 on cell proliferation and apoptosis. A The effect of S100A9 on A431 cell proliferation was determined by CCK-8. B The effect of S100A9 on A431 cell apoptosis was measured by staining with Annexin V/PI, followed by FACS analysis. C The effect of $F A B P 5$ on $A 431$ cell proliferation was determined by CCK-8. D The effect of FABP5 on A431 cell apoptosis was measured by staining with Annexin V/PI, followed by FACS analysis. E The effect of S100A9 and FABP5 on SCL-1 cell proliferation. F The effect of S100A9 and FABP5 on SCL1 cell apoptosis. Data were presented as mean \pm SD. siNC represents the siRNA negative control, and siS100A9 and siFABP5 represent the specific S100A9 siRNA and FABP5 siRNA, respectively.

\section{CONCLUSION}

In conclusion, our findings provide valuable resources for deciphering comprehensive gene expression landscapes of heterogeneous cell types in CSCC. In specific, many members from S100 gene family, SPRR gene family, and FABP5 were highly expressed in CSCC cells. SOOA9 and FABP5 influence cell proliferation and migration through NF-KB pathway. Thus, these results suggested that targeting S100A9 or 
A

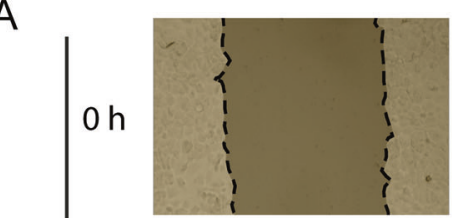

$24 \mathrm{~h}$

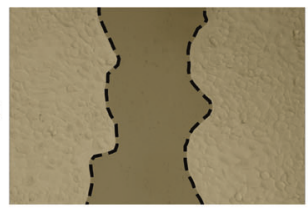

siNC

$\frac{5}{4}$

$\mathrm{oh}$

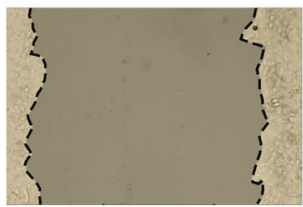

$24 \mathrm{~h}$

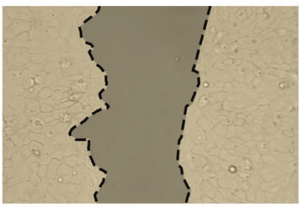

siNC

B

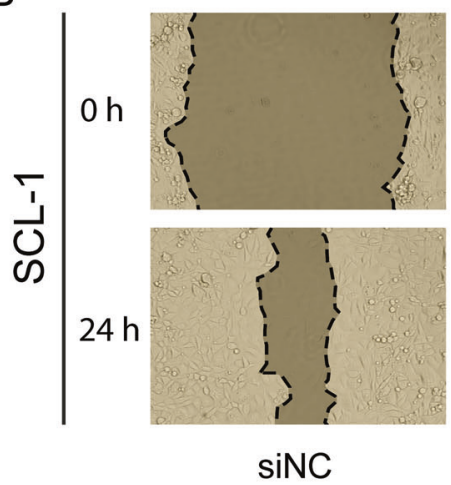

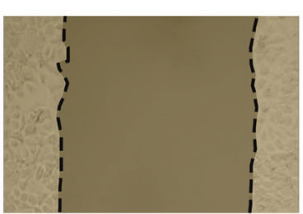

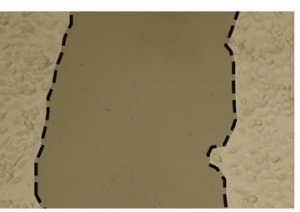

siS100A9
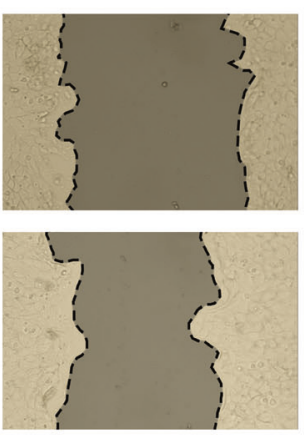

siFABP5
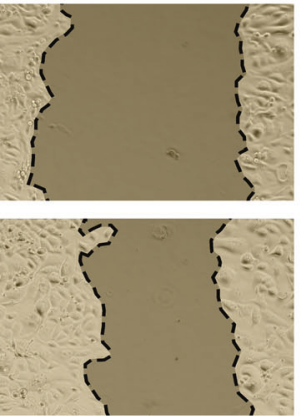

siS100A9
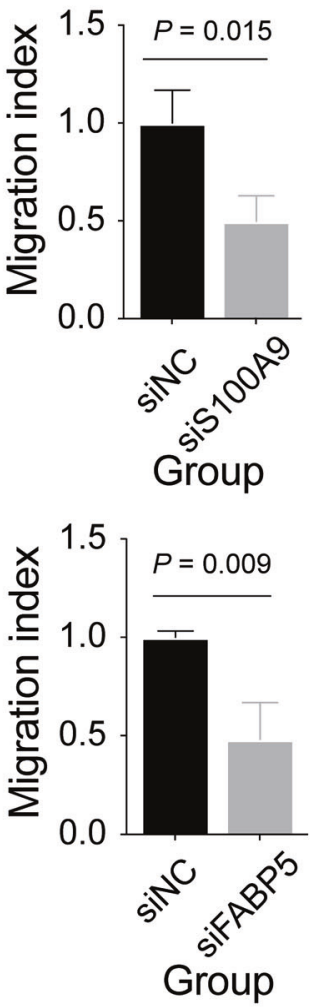

Group

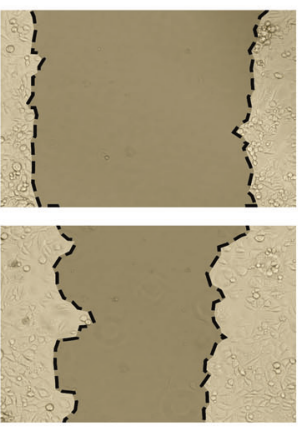

SiFABP5
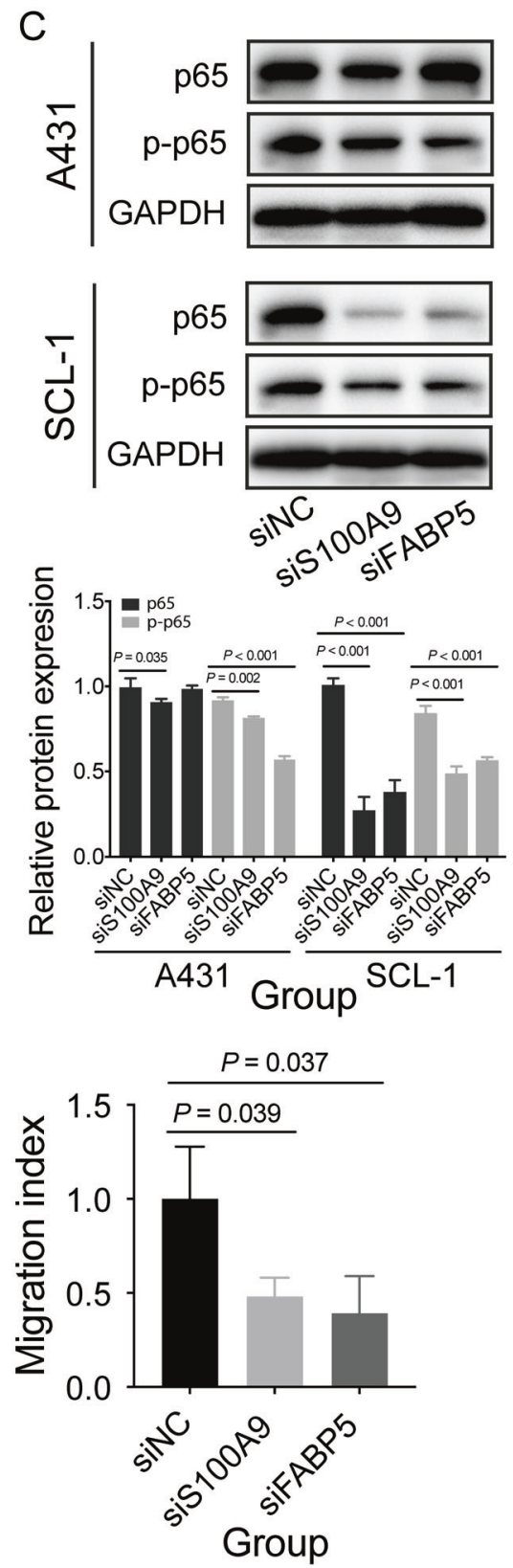

Fig. 7 Effect of S100A9 and FABP5 on cell migration and NF-KB pathway. A, B The effect of S100A9 and FABP5 on cell migration and invasion were determined by wound-healing assay. C The effect of S100A9 and FABP5 on NF-KB pathway were determined by western blot analysis. Data were presented as mean \pm SD. siNC represents the siRNA negative control, and siS100A9 and siFABP5 represent the specific S100A9 siRNA and FABP5 siRNA, respectively. The statistical method is the unpaired Student's $t$-test.

FABP5 might be an effective approach for suppressing cSCC progression.

\section{DATA AVAILABILITY}

The data that support the findings of this study are available from the corresponding author upon reasonable request as another research is being conducted.

\section{CODE AVAILABILITY}

The complete computational analysis workflow of this study is available from the corresponding author upon reasonable request.

\section{REFERENCES}

1. Global Burden of Disease Cancer Collaboration, Fitzmaurice C, Abate D, Abbasi $N$, Abbastabar $\mathrm{H}$, Abd-Allah F, et al. Global, regional, and national cancer incidence, mortality, years of life lost, years lived with disability, and disability-adjusted lifeyears for 29 cancer groups, 1990 to 2017: a systematic analysis for the Global Burden of Disease study. JAMA Oncol. 2019;5:1749-68.

2. Sung H, Ferlay J, Siegel RL, Laversanne M, Soerjomataram I, Jemal A, et al. Global cancer statistics 2020: GLOBOCAN estimates of incidence and mortality worldwide for 36 cancers in 185 countries. CA Cancer J Clin. 2021;71:209-49.

3. Thompson AK, Kelley BF, Prokop LJ, Murad MH, Baum CL. Risk factors for cutaneous squamous cell carcinoma recurrence, metastasis, and disease-specific death: a systematic review and meta-analysis. JAMA Dermatol. 2016;152:419-28.

4. Karia PS, Han J, Schmults CD. Cutaneous squamous cell carcinoma: estimated incidence of disease, nodal metastasis, and deaths from disease in the United States, 2012. J Am Acad Dermatol. 2013;68:957-66. 
5. Janus JM, O'Shaughnessy RFL, Harwood CA, Maffucci T. Phosphoinositide 3kinase-dependent signalling pathways in cutaneous squamous cell carcinomas. Cancers (Basel). 2017;9:86.

6. Ratushny V, Gober MD, Hick R, Ridky TW, Seykora JT. From keratinocyte to cancer: the pathogenesis and modeling of cutaneous squamous cell carcinoma. J Clin Invest. 2012;122:464-72.

7. Migden MR, Rischin D, Schmults CD, Guminski A, Hauschild A, Lewis KD, et al. PD1 blockade with cemiplimab in advanced cutaneous squamous-cell carcinoma. $\mathrm{N}$ Engl J Med. 2018;379:341-51.

8. Grob JJ, Gonzalez R, Basset-Seguin N, Vornicova O, Schachter J, Joshi A, et al. Pembrolizumab monotherapy for recurrent or metastatic cutaneous squamous cell carcinoma: a single-arm phase II trial (KEYNOTE-629). J Clin Oncol. 2020;38:2916-25

9. Maubec E, Boubaya M, Petrow P, Beylot-Barry M, Basset-Seguin N, Deschamps L, et al. Phase II study of pembrolizumab as first-line, single-drug therapy for patients with unresectable cutaneous squamous cell carcinomas. J Clin Oncol. 2020;38:3051-61.

10. Lazar AD, Dinescu S, Costache M. Deciphering the molecular landscape of cutaneous squamous cell carcinoma for better diagnosis and treatment. J Clin Med. 2020;9:2228.

11. Kim D, Chung KB, Kim TG. Application of single-cell RNA sequencing on human skin: technical evolution and challenges. J Dermatol Sci. 2020;99:74-81.

12. van der Wijst MGP, Brugge $H$, de Vries DH, Deelen $P$, Swertz MA, LifeLines Cohort $\mathrm{S}$, et al. Single-cell RNA sequencing identifies celltype-specific cis-eQTLs and coexpression QTLs. Nat Genet. 2018;50:493-7.

13. Miao $Y$, Yang $H$, Levorse J, Yuan S, Polak L, Sribour $M$, et al. Adaptive immune resistance emerges from tumor-initiating stem. Cells Cell. 2019;177:1172-86. e1114.

14. Yost KE, Satpathy AT, Wells DK, Qi Y, Wang C, Kageyama R, et al. Clonal replacement of tumor-specific T cells following PD-1 blockade. Nat Med. 2019;25:1251-9.

15. Ji AL, Rubin AJ, Thrane K, Jiang S, Reynolds DL, Meyers RM, et al. Multimodal analysis of composition and spatial architecture in human squamous. Cell Carcinoma Cell. 2020;182:497-514.e422.

16. Tirosh I, Izar B, Prakadan SM, Wadsworth MH II, Treacy D, Trombetta JJ, et al. Dissecting the multicellular ecosystem of metastatic melanoma by single-cell RNA-seq. Science. 2016;352:189-96.

17. Picelli S, Bjorklund AK, Faridani OR, Sagasser S, Winberg G, Sandberg R. Smartseq2 for sensitive full-length transcriptome profiling in single cells. Nat Methods. 2013;10:1096-8.

18. Andrews S. FastQC: a quality control tool for high throughput sequence data. 2010.

19. Bolger AM, Lohse $M$, Usadel B. Trimmomatic: a flexible trimmer for Illumina sequence data. Bioinformatics. 2014;30:2114-20.

20. Pertea M, Kim D, Pertea GM, Leek JT, Salzberg SL. Transcript-level expression analysis of RNA-seq experiments with HISAT, StringTie and Ballgown. Nat Protoc. 2016;11:1650-67

21. Liao Y, Smyth GK, Shi W. featureCounts: an efficient general purpose program for assigning sequence reads to genomic features. Bioinformatics. 2014;30:923-30.

22. Lun AT, McCarthy DJ, Marioni JC. A step-by-step workflow for low-level analysis of single-cell RNA-seq data with bioconductor. F1000Res. 2016;5:2122.

23. Stuart T, Butler A, Hoffman P, Hafemeister C, Papalexi E, Mauck WM III, et al. Comprehensive integration of single-cell data. Cell. 2019;177:1888-902.e1821.

24. Zhang G, Yan G, Fu Z, Wu Y, Wu F, Zheng Z, et al. Loss of retinoic acid receptorrelated receptor alpha (Roralpha) promotes the progression of UV-induced CSCC. Cell Death Dis. 2021;12:247.

25. Jamal-Hanjani M, Wilson GA, McGranahan N, Birkbak NJ, Watkins TBK, Veeriah S, et al. Tracking the evolution of non-small-cell lung cancer. $\mathrm{N}$ Engl J Med. 2017;376:2109-21.

26. Saunders NA, Simpson F, Thompson EW, Hill MM, Endo-Munoz L, Leggatt G, et al. Role of intratumoural heterogeneity in cancer drug resistance: molecular and clinical perspectives. EMBO Mol Med. 2012;4:675-84.

27. McGranahan N, Swanton C. Clonal heterogeneity and tumor evolution: past, present, and the future. Cell. 2017;168:613-28.

28. Zhang $Y$, Wang D, Peng M, Tang L, Ouyang J, Xiong F, et al. Single-cell RNA sequencing in cancer research. J Exp Clin Cancer Res. 2021;40:81.

29. Lahnemann D, Koster J, Szczurek E, McCarthy DJ, Hicks SC, Robinson MD, et al. Eleven grand challenges in single-cell data science. Genome Biol. 2020;21:31.

30. Dotto GP. Multifocal epithelial tumors and field cancerization: stroma as a primary determinant. J Clin Invest. 2014;124:1446-53.

31. Bresnick AR, Weber DJ, Zimmer DB. S100 proteins in cancer. Nat Rev Cancer. 2015;15:96-109.

32. Foell D, Roth J. Proinflammatory S100 proteins in arthritis and autoimmune disease. Arthritis Rheum. 2004;50:3762-71.
33. Gebhardt C, Nemeth J, Angel P, Hess J. S100A8 and S100A9 in inflammation and cancer. Biochem Pharm. 2006;72:1622-31.

34. Wang J, Cai Y, Xu H, Zhao J, Xu X, Han YL, et al. Expression of MRP14 gene is frequently down-regulated in Chinese human esophageal cancer. Cell Res. 2004;14:46-53.

35. Choi DK, Li ZJ, Chang IK, Yeo MK, Kim JM, Sohn KC, et al. Clinicopathological roles of S100A8 and S100A9 in cutaneous squamous cell carcinoma in vivo and in vitro. Arch Dermatol Res. 2014;306:489-96.

36. Shin JM, Chang IK, Lee YH, Yeo MK, Kim JM, Sohn KC, et al. Potential role of S100A8 in cutaneous squamous cell carcinoma differentiation. Ann Dermatol. 2016:28:179-85.

37. Ichikawa M, Williams R, Wang L, Vogl T, Srikrishna G. S100A8/A9 activate key genes and pathways in colon tumor progression. Mol Cancer Res. 2011;9:133-48.

38. Cabral A, Voskamp P, Cleton-Jansen AM, South A, Nizetic D, Backendorf C. Structural organization and regulation of the small proline-rich family of cornified envelope precursors suggest a role in adaptive barrier function. J Biol Chem. 2001;276:19231-7.

39. Mizuguchi Y, Isse K, Specht S, Lunz JG III, Corbitt N, Takizawa T, et al. Small proline rich protein $2 a$ in benign and malignant liver disease. Hepatology. 2014;59:1130-43.

40. Vermeij WP, Backendorf C. Skin cornification proteins provide global link between ROS detoxification and cell migration during wound healing. PLoS One. 2010;5:e11957.

41. Li X, Mohan S, Gu W, Baylink DJ. Analysis of gene expression in the wound repair/ regeneration process. Mamm Genome. 2001;12:52-59.

42. De Craene B, Berx G. Regulatory networks defining EMT during cancer initiation and progression. Nat Rev Cancer. 2013;13:97-110.

43. Shirkoohi R. Epithelial mesenchymal transition from a natural gestational orchestration to a bizarre cancer disturbance. Cancer Sci. 2013;104:28-35.

44. Zhang $\mathrm{Y}$, Wang $\mathrm{H}$, Wang J, Bao L, Wang L, Huo J, et al. Global analysis of chromosome 1 genes among patients with lung adenocarcinoma, squamous carcinoma, large-cell carcinoma, small-cell carcinoma, or non-cancer. Cancer Metastasis Rev. 2015;34:249-64.

45. Nisa L, Barras D, Medova M, Aebersold DM, Medo M, Poliakova M, et al. Com prehensive genomic profiling of patient-matched head and neck cancer cells: a preclinical pipeline for metastatic and recurrent disease. Mol Cancer Res. 2018;16:1912-26.

46. Wang Y, Wang L, Li X, Qu X, Han N, Ruan M, et al. Decreased CSTA expression promotes lymphatic metastasis and predicts poor survival in oral squamous cell carcinoma. Arch Oral Biol. 2021;126:105116.

47. Luo A, Kong J, Hu G, Liew CC, Xiong M, Wang X, et al. Discovery of Ca2+-relevant and differentiation-associated genes downregulated in esophageal squamous cell carcinoma using cDNA microarray. Oncogene. 2004;23:1291-9.

48. Xu X, Wei S, Chen Y, Yu D, Wang X, Dong X. Serum small proline-rich protein 2A (SPRR2A) is a noninvasive biomarker in gastric cancer. Dis Markers. 2020;2020:8493796.

49. Specht S, Isse K, Nozaki I, Lunz JG III, Demetris AJ. SPRR2A expression in cholangiocarcinoma increases local tumor invasiveness but prevents metastasis. Clin Exp Metastasis. 2013;30:877-90.

50. Mizuguchi Y, Specht S, Lunz JG III, Isse K, Corbitt N, Takizawa T, et al. SPRR2A enhances $\mathrm{p} 53$ deacetylation through HDAC1 and down regulates p21 promoter activity. BMC Mol Biol. 2012;13:20.

51. Bian X, Liu R, Meng Y, Xing D, Xu D, Lu Z. Lipid metabolism and cancer. J Exp Med. 2021;218:e20201606.

52. Ogawa $E$, Owada $Y$, lkawa $S$, Adachi $Y$, Egawa $T$, Nemoto $K$, et al. Epidermal FABP (FABP5) regulates keratinocyte differentiation by $13(\mathrm{~S})$-HODE-mediated activation of the NF-kappaB signaling pathway. J Invest Dermatol. 2011;131:604-12.

53. Owada Y, Suzuki I, Noda T, Kondo H. Analysis on the phenotype of E-FABP-gene knockout mice. Mol Cell Biochem. 2002;239:83-86.

54. Watanabe $\mathrm{R}$, Fujii $\mathrm{H}$, Yamamoto $A$, Hashimoto $T$, Kameda $K$, Ito $M$, et al. Immunohistochemical distribution of cutaneous fatty acid-binding protein in human skin. J Dermatol Sci. 1997;16:17-22.

55. Masouye I, Saurat JH, Siegenthaler G. Epidermal fatty-acid-binding protein in psoriasis, basal and squamous cell carcinomas: an immunohistological study. Dermatology. 1996;192:208-13.

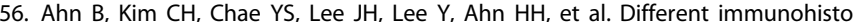
chemical localization of fatty acid binding protein 5 in actinic keratosis compared with that in Bowen's disease: A retrospective study. Am J Dermatopathol. 2021:43:356-61.

57. Fang LY, Wong TY, Chiang WF, Chen YL. Fatty-acid-binding protein 5 promotes cell proliferation and invasion in oral squamous cell carcinoma. J Oral Pathol Med. 2010;39:342-8.

58. Zhang $C$, Liao $Y$, Liu $P$, Du $Q$, Liang $Y$, Ooi $S$, et al. FABP5 promotes lymph node metastasis in cervical cancer by reprogramming fatty acid metabolism. Theranostics. 2020;10:6561-80. 
12

59. Senga S, Kobayashi N, Kawaguchi K, Ando A, Fujii H. Fatty acid-binding protein 5 (FABP5) promotes lipolysis of lipid droplets, de novo fatty acid (FA) synthesis and activation of nuclear factor-kappa B (NF-kappaB) signaling in cancer cells. Biochim Biophys Acta Mol Cell Biol Lipids. 2018;1863:1057-67.

60. Seo J, Jeong DW, Park JW, Lee KW, Fukuda J, Chun YS. Fatty-acid-induced FABP5/ HIF-1 reprograms lipid metabolism and enhances the proliferation of liver cancer cells. Commun Biol. 2020;3:638.

61. Hagens G, Masouye I, Augsburger E, Hotz R, Saurat JH, Siegenthaler G. Calciumbinding protein $\mathrm{S} 100 \mathrm{~A} 7$ and epidermal-type fatty acid-binding protein are associated in the cytosol of human keratinocytes. Biochem J. 1999;339:419-27.

\section{ACKNOWLEDGEMENTS}

This research was financially funded by the National Natural Science Foundation of China (81872212, 82073013, 82073016, and 81801862), the General Project of Shanghai Municipal Science and Technology Commission (20ZR1449400), and Shanghai Municipal Science and Technology Commission (19441904500).

\section{AUTHOR CONTRIBUTIONS}

Conceptualization: GZ, YT, and XW; methodology: GY, LL, and SZ; validation: LZ, NJ, and $\mathrm{SZ}$; formal analysis: $\mathrm{GY}$ and $\mathrm{LL}$; investigation: $\mathrm{ZZ}, \mathrm{FW}, \mathrm{CL}$, and LJ; resources: $\mathrm{YL}, \mathrm{QZ}$, $P W$, and LS; data curation: YW, ZZ, and SF; writing - original draft preparation: GY, LL, and $S Z$; writing - review and editing: $G Z, Y T$, and $X W$; visualization: $G Y$; supervision: $\mathrm{GZ}, \mathrm{YT}$, and XW; project administration: XW; funding acquisition: SF, SZ, GZ, and XW. All authors have read and agreed to the published version of the manuscript.

\section{COMPETING INTERESTS}

The authors declare no competing interests.

\section{ETHICS APPROVAL}

All patients provided were consented preoperatively to enroll in this study, which was reviewed and approved by the Medical Ethics Committee of Shanghai Skin
Disease Hospital. All samples were used according to the ethical guidelines of the 1975 Declaration of Helsinki and obtained with the patients' understanding that it might be published.

\section{ADDITIONAL INFORMATION}

Supplementary information The online version contains supplementary material available at https://doi.org/10.1038/s41419-021-04477-y.

Correspondence and requests for materials should be addressed to Guolong Zhang, Yichen Tang or Xiuli Wang.

Reprints and permission information is available at http://www.nature.com/ reprints

Publisher's note Springer Nature remains neutral with regard to jurisdictional claims in published maps and institutional affiliations. Attribution 4.0 International License, which permits use, sharing, adaptation, distribution and reproduction in any medium or format, as long as you give appropriate credit to the original author(s) and the source, provide a link to the Creative Commons license, and indicate if changes were made. The images or other third party material in this article are included in the article's Creative Commons license, unless indicated otherwise in a credit line to the material. If material is not included in the article's Creative Commons license and your intended use is not permitted by statutory regulation or exceeds the permitted use, you will need to obtain permission directly from the copyright holder. To view a copy of this license, visit http://creativecommons. org/licenses/by/4.0/

(c) The Author(s) 2021 